\title{
Fatal anaphylactoid reaction following ioversol administration
}

\author{
Frank GA Jansman · Hans Kieft · Johannes W Harting
}

Received: 20 October 2006/Accepted: 25 March 2007/Published online: 8 June 2007

(C) Springer Science+Business Media B.V. 2007

\begin{abstract}
We report a fatal intravenous ioversol administration in a 60-year old male patient. Although the introduction of new low-osmolar non-ionogenic contrast media with a more favourable efficacy-toxicity balance has diminished the side-effects significantly, everyone involved in radiodiagnostic procedures should be aware of the potential life-threatening effects. Especially patients with risk factors for side-effects should be monitored carefully.
\end{abstract}

Keywords Contrast media - Anaphylactoid reaction . Adverse effects · Risk factors

\section{Introduction}

For several decades, organic iodinated contrast media have been used for diagnostic radiologic imaging purposes. Because of the absorption difference of X-rays by the iodine-molecule and surrounding tissues, radiographic visualisation of structures is possible. Although new compounds with a more favourable efficacy-toxicity balance have been introduced, severe and life-threatening side-effects are still reported.

F. G. Jansman · J. W. Harting

Department of Clinical Pharmacy, Isala klinieken, Zwolle,

The Netherlands

F. G. Jansman ( $₫)$

Department of Pharmacotherapy and Pharmaceutical Care, Groningen University Institute for Drug Exploration (GUIDE),

Antonius Deusinglaan 1, 9713 AV Groningen, The Netherlands

e-mail: f.g.a.jansman@isala.nl

H. Kieft

Department of Intensive Care, Isala klinieken, Zwolle,

The Netherlands
We present a male patient with a fatal cardiac arrest after intravenous ioversol administration.

\section{Case description}

A 60-year-old man, with a history of regulated diabetes mellitus type II and hypertension, was referred for an abdominal contrast-enhanced CT-scan, because of presurgical staging of a rectal carcinoma His medication consisted of metformin and a thiazide diuretic. There was no history of allergic reactions.

After ioversol 350, $100 \mathrm{ml}$ had been administered intravenously, the patient complained of headache and nausea. Subsequently, he lost consciousness with discoloration of his skin to red-purple. Immediate basic life support was started. On arrival of the medical emergency team, patient was found in pulseless electrical activity. During endotracheal intubation no signs of glottis edema were noticed. Ventricular fibrillation developed for which (unsuccessful) external electrical defibrillation was performed. After 40 min of cardiopulmonary resuscitation with repeated doses of epinephrine, clemastine, atropine, and amiodarone, with concomitant dexamethasone, calcium, theophylline, transcutaneous pacing and intracardial epinephrine, the transthoracic echocardiogram yielded cardiac standstill without ventricular dilatation. It was then decided to cease resuscitation.

An autopsy found, besides the rectal carcinoma without lymph node metastasis, benign adrenal and prostatic hyperplasia concentric left ventricular hypertrophy.

\section{Discussion}

Ioversol, used in the present case, is a low-osmolar nonionic monomeric contrast medium, with a relatively 
low risk of (severe) side-effects. According to the information from the manufacturer, anaphylactoid reactions occur in $0.1-1 \%$ of cases, life-threatening in less than $0.01 \%$ [1]. The Netherlands Pharmacovigilance Centre (LAREB) received no report of pseudo-allergic reactions to ioversol administration since its registration in 2001, although one report of severe dyspnoe was found [2].

Intravenous administration of low-osmolar contrast media is associated with side-effects in $2.1 \%$ of patients, being mild in $1.2 \%$, moderate in $0.9 \%$, and severe in $0.01 \%$ [3]. For the $52 \%$ of patients in this study that had risk factors for adverse effects, not being defined in detail, the side effect rate was $2.7 \%$.

The toxicity of contrast media is determined by the intrinsic toxicity and the osmolarity of the medium.

The intrinsic toxicity is attributed to the ability to bind to biological macro-molecules, such as proteins [4]. Since non-ionic and dimeric contrast molecules bind to proteins to a lesser extent than ionic and monomeric contrast molecules, contrast media with non-ionic dimeric molecules (like ioversol) are less toxic.

Life-threatening hypersensitivity reactions have been reported on intravenous administration of iodinated contrast media [5]. Most frequent symptoms are fever, purpura, skin reactions, cardiovascular collapse (like in the present case), convulsions, dyspnoe, renal impairment, pseudo-allergic and/or anaphylactoid reactions [1]. In contrast to initial exposure, clinically manifest IgE-reactions arise at renewed exposure to the allergenic agent. Therefore, anaphylactic reactions to iodinated contrast media are specified as non-allergic anaphylactic or anaphylactoïd reactions. The clinical symptoms, however, of allergic and non-allergic anaphylactic reactions are similar [6].

Several risk factors are identified for the occurrence of side-effects of iodinated contrast media [1] (Table 1); in the present case, the patient had the following risk factors: diabetes, cardiovascular disease and co-medication (metformine). Recommendations for the prevention of renal side-effects are well known and include the preferred use of low-osmolar over high-osmolar contrast media, avoidance of the administration of high doses, sufficient hydration, and prophylactic administration of antioxidants in order to disable the action of cytotoxic oxygen free radicals causing ischemic renal injury. Moreover, calcium-channel blockers can be considered for their positive effects on renal haemodynamics and their cytoprotective effects on renal cells. Furthermore, pre-heating of the contrast fluid before administration decreases its viscosity, reducing side-effects as well [7].
Table 1 Risk factors for side-effects of contrast media

Previous reactions on iodinated contrast media

Positive allergic anamnesis

High osmolar contrast medium

High dose contrast medium (>100 ml)

Intra-arterial injection

Dehydration

Cardiovascular disease, e.g. congestive heart failure, hypertension

Pre-existing renal impairment

Diabetes mellitus

Hyperuricaemia

Proteinuria

Nephrotoxic co-medication (e.g. metformine, vasopressor drugs, nonsteroidal anti-inflammatory drugs, diuretics, aminoglycosides)

Co-medication (neuroleptic drugs, antidepressants, analeptic drugs, MAO-inhibitors)

Hyperthyroidism

Severe hepatic impairment/liver cirrhosis and necrosis

Pheochromocytoma

Cerebro-vascular disease

Homocystinuriae

Sickel cell anaemia

Uraemia

Morbus Kahler

Epilepsy

Alcoholism/liver cirrhosis and necrosis

Asthma

Drug addiction

Age $>70$ years

\section{Conclusion}

In the present case, ioversol administration evidently caused the death of this patient. Despite adequate intervention of the medical emergency team, cardiopulmonary resuscitation was not successful. Although the introduction of low-osmolar noniogenic contrast media has diminished the side-effects significantly, every radiologist and others involved in radiodiagnostic procedures should be aware of the potential life-threatening effects. Especially patients with risk factors for side-effects, including cardiovascular disease, diabetes, renal impairment, and particular comedication, should be monitored carefully with an adequately organized and operating medical emergency team on duty. Appropriate guidelines for the treatment of acute adverse reactions and equipment available in the room where the contrast medium is given, are provided by the Contrast Media Safety Committee of the European Society of Urogenital Radiology and the American College of Radiology [8, 9]. 


\section{References}

1. Optiray [product information]. Petten, the Netherlands: Mallinckrodt Medical BV; 2004.

2. Adverse Drug Reaction Database. 's Hertogenbosch, the Netherlands: Netherlands Pharmacovigilance Centre Lareb. Available at: http://www.lareb.nl. Accessed February 8th, 2007.

3. Schrott KM, Behrends B, Clauss W, Kaufmann J, Lehnert J. Iohexol in excretory urography: results of the drug monitoring programme. Fortsch Med 1986; 104:153-6.

4. Morcos SK, Dawson P, Pearson JD, et al. The haemodynamic effects of iodinated water soluble radiographic contrast media: a review. Eur J Radiol 1998; 29:31-46.
5. Pennington JAT. A review of iodine toxicity reports. J Am Diet Assoc 1990; 90:1571-81.

6. Murrant T, Bihari D. Anaphylaxis and anaphylactoid reactions. Int J Clin Pract 2000; 54:322-8.

7. Torvik A, Walday P. Neurotoxicity of water-soluble contrast media. Acta Radiol 1995; 36:221-9.

8. Thomsen HS, Morcos SK. Contrast Media Safety Committee of European Society of Urogenital Radiology. Management of acute adverse reactions to contrast media. Eur Radiol 2004; 14:476-81.

9. American College of Radiology. Manual on contrast media, version 5.0. Accessed February 8th, 2007, at http://www.acr.org/ s_acr/sec.asp?CID $=2131 \&$ DID $=16687$. 